\title{
Simulator Evaluation of the Final Approach Spacing Tool
}

Thomas J. Davis, Heinz Erzberger, and Steven M. Green

May 1990

(NASA-TH-1O2AOT) BTULATOZ EVALUATTONOF

THE WTAL APPROAH SPACING TUOL (NASA)

OSL OLC

$100-23374$

$$
63103 \quad 0287436
$$


NASA Technical Memorandum 102807

\section{Simulator Evaluation of the Final Approach Spacing Tool}

Thomas J. Davis, Heinz Erzberger, and Steven M. Green Ames Research Center, Moffett Field, California

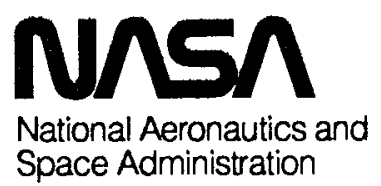




\section{TABLE OF CONTENTS}

Page

SUMMARY …

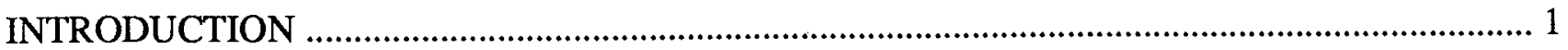

OVERVIEW OF AUTOMATION SYSTEM CONCEPT …………................................................ 2

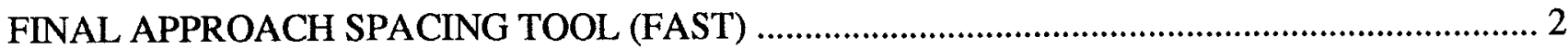

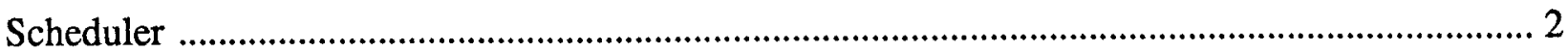

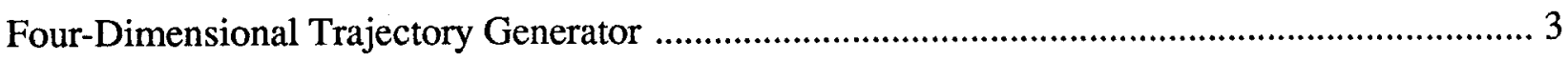

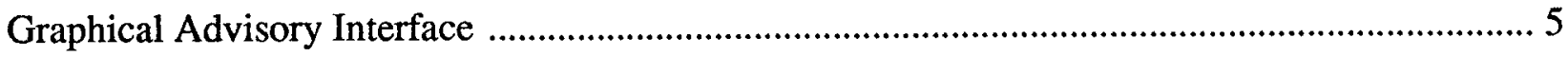

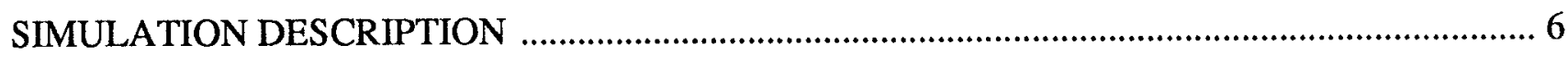

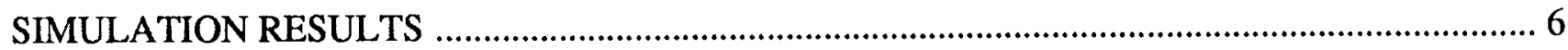

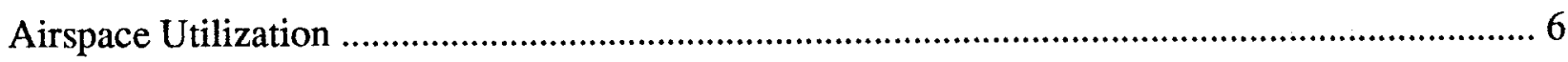

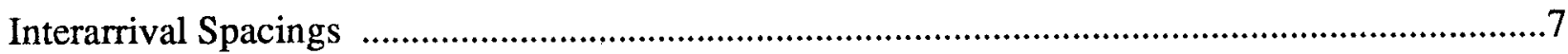

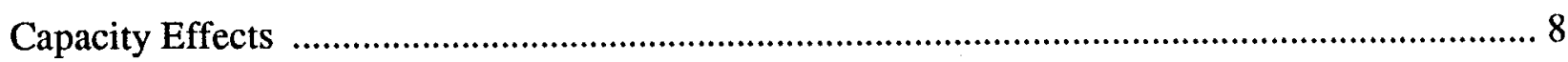

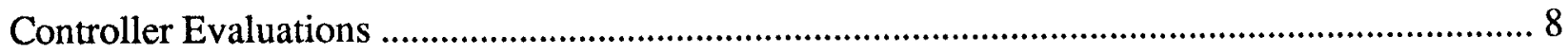

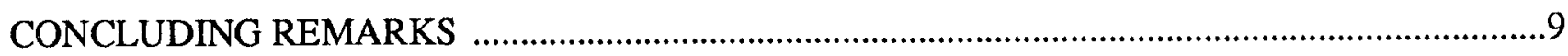

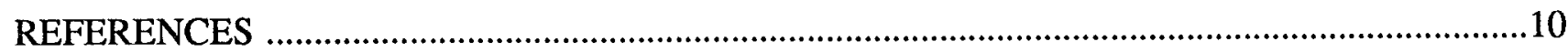

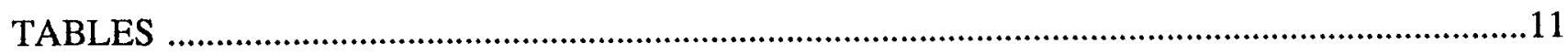

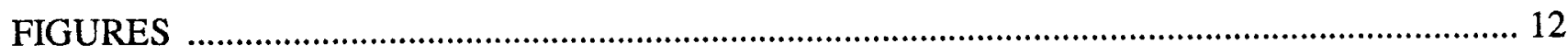




\section{SUMMARY}

This paper describes the design and simulator evaluation of an automation tool for assisting terminal radar approach controllers in sequencing and spacing traffic onto the final approach course. The automation tool, referred to as the Final Approach Spacing Tool (FAST), displays speed and heading advisories for arrivals as well as sequencing information on the controller's radar display. The main functional elements of FAST are a scheduler that schedules and sequences the traffic, a 4D trajectory synthesizer that generates the advisories, and a graphical interface that displays the information to the controller. FAST has been implemented on a high performance workstation. It can be operated as a standalone in the Terminal Radar Approach Control (TRACON) Facility or as an element of a system integrated with automation tools in the Air Route Traffic Control Center (ARTCC). FAST was evaluated by experienced TRACON controllers in a real-time air traffic control simulation. Simulation results summarized in the paper show that FAST significantly reduced controller workload and demonstrated a potential for an increase in landing rate.

\section{INTRODUCTION}

Increasing delays and airspace congestion at major airports are among the most critical problems facing the air transportation system. It is widely recognized that the introduction of advanced automation techniques in air traffic control (ATC) offers a high potential for alleviating these problems. This paper describes the design of an automation system for assisting controllers in the management of arrival traffic in the terminal area.

The first innovative design of an automation system for terminal area ATC was developed in the late 1960s (ref. 1). This system, the progenitor of all automation aids, provided speed and heading advisories to help controllers increase spacing accuracy on final approach. Although traffic tests of the system showed an increase in landing rate, controllers found their workload was increased and they rejected the system. A retrospective examination of the concept suggests that the design was sound but its effectiveness was limited by the technology of the period, especially its lack of an adequate controller interface. A recent fast time simulation study confirmed the potential for increasing landing rate with the assistance of such automation aids (ref. 2).

Recent research at NASA Ames has resulted in the design and laboratory implementation of an integrated Center-TRACON Automation System for the efficient control of arrival traffic. The elements comprising this system are the Traffic Management Advisor (TMA) and the Descent Advisor (DA) to be used in Air Route Traffic Control Centers (ARTCC or Center) and the Final Approach Spacing Tool (FAST) to be used in Terminal Radar Approach Control (TRACON) Facilities (refs. 3, 4). The advisories generated by these tools assist controllers in handling aircraft arrivals starting at about 200 n.mi. ( $45 \mathrm{~min})$ from the airport and continuing to the final approach fix. During the last two years, the three elements of this system have been evaluated by Center and TRACON controllers in several real-time simulations. 
This paper begins with an overview of the Center-TRACON Automation System tools (i.e., the TMA, DA, and FAST). Then the paper focuses on the design and evaluation of FAST, the main function of which is to provide speed and turn advisories that help controllers achieve an accurately spaced flow of traffic on final approach. The paper concludes with a description of results from a recent real-time simulation which evaluated the acceptability of FAST to TRACON controllers and its effect on landing rate.

\section{OVERVIEW OF AUTOMATION SYSTEM CONCEPT}

The Center-TRACON Automation System consists of three sets of integrated tools, referred to as TMA, DA, and FAST. TMA is a tool for the Center whose primary function is to plan the most efficient landing times. The scheduling algorithms implemented in TMA generate landing sequences that minimize overall system delay. The TMA plans these times such that traffic approaching from different directions will merge on the final approach without conflicts and with optimal spacing. The principal function of the DA is to assist the Center controllers in implementing the arrival schedules generated by the TMA. It provides descent speed profiles, top of descent points, and turn advisories that help controllers deliver aircraft at feeder gates into the TRACON at specified times. DA also provides conflict detection and resolution advisories, together with an interactive graphical interface. A detailed description of the TMA and DA is given in reference 3 .

FAST provides tools to assist TRACON controllers in keeping aircraft on precise time controlled trajectories from the feeder gates (the entry point into the TRACON) to the final approach fix. Although primarily based on the same set of scheduling and 4D trajectory algorithms as the Center TMA and DA, it also has several capabilities designed specifically to handle the unique problems occurring in the TRACON. A review of the principal design features of FAST can be found in reference 4; they are summarized in the following sections.

\section{FINAL APPROACH SPACING TOOL (FAST)}

The Final Approach Spacing Tool consists of three major software elements: a scheduler, a 4D trajectory generator, and a graphical advisory interface, each of which is briefly described below.

\section{Scheduler}

The function of the Scheduler incorporated in FAST is to generate optimally spaced landing times for arrival aircraft. These landing times are subsequently fed as input to the 4D trajectory generator in FAST, which computes appropriate heading and speed advisories that help the controller keep the aircraft on time. The scheduling algorithm in FAST is essentially identical to the one in the Center TMA. The primary difference between them involves the choice of the Scheduling and Freeze Horizons. These time parameters determine when arrivals are initially assigned landing times and when the landing times

are frozen. Appropriate values for these parameters were determined experimentally and are typically set at $11 \mathrm{~min}$ and $8 \mathrm{~min}$ to touchdown, respectively. 
The operation of the Scheduler, described in references 3 and 5 is briefly reviewed here. The primary inputs to the Scheduler are periodically updated estimated times of arrival (ETAs) for all aircraft that are being tracked by the terminal area radar systems. When the ETA of a new arrival first falls within the Scheduling Window, which is defined as the time interval between the Scheduling and Freeze Horizons, the Scheduler begins generating scheduled times of arrival (STAs). The Scheduler first attempts to place a new arrival at a time identical to its ETA on the runway. If such a choice of STA creates a spacing violation with previously scheduled aircraft, the Scheduler assigns the closest available time that meets the minimum allowed spacing distance on final approach. The minimum time separations used by the Scheduler are derived from minimum separation distances specified by FAA regulations. The minimum spacing distances depend on the weight classes of the aircraft in the landing sequence and can be represented in a matrix of separation distances (n.mi.) as given in table 1. As explained in reference 6, this matrix of distances is converted to a corresponding matrix of time separations by incorporating knowledge of final approach speeds. Furthermore, buffers on the order of 10 to $20 \mathrm{sec}$ are added to these minimum time separations in order to protect against unavoidable errors in the ability to control landing times using the FAST advisories.

The magnitude of the differences between the STAs and the initial ETAs generated by the Scheduler depends both on the orderliness of the arrival stream and on the excess of the total arrival flow over the maximum landing rate. If the arrivals into the TRACON airspace are controlled by the DA and TMA, they will arrive at the gates with only small time errors and the flow rate will match the runway acceptance rate. In that case the Scheduler in FAST will make only minor changes in the STAs originally calculated by the Center TMA. These changes will correct the small time error accumulated during the descent and the transition from the Center into the TRACON. Most of the time, therefore, the Scheduler will be able to preserve the optimal landing sequence originally calculated by the Center TMA.

If the Center automation tools, DA and TMA, are not in operation, the flow into the TRACON during rush periods will be strongly bunched and may exceed the maximum runway acceptance rate for a period of time. Because of maneuver airspace restrictions and other factors, a TRACON Scheduler has less freedom to optimize the arrival sequence than the Center Scheduler, and therefore cannot be as effective in reducing delays. However, the FAST scheduler is designed to handle such difficult flow conditions in the best possible way. It will generate landing sequences and STAs that minimize delays subject to operational constraints. Under excess traffic load, the STAs generated by the Scheduler will absorb delays in the TRACON by holding or path stretching.

An important function built into the Scheduler is the capability for handling missed approaches and popup traffic. With these functions, the Scheduler opens up a time slot where such aircraft can be reinserted into the arrival sequence. Under saturated traffic conditions the insertion of an extra slot will, inevitably, introduce delays for aircraft that follow the inserted aircraft. The rescheduling function assists the controller in finding a slot in the arrival sequence that will least disrupt the overall traffic flow.

\section{Four-Dimensional Trajectory Generator}

The FAST descent trajectory synthesis algorithm is a modified version of the Center DA algorithm. A detailed description of the algorithm is given in reference 3 . Similar to the Center DA, it employs a 
second-order Runge-Kutta forward integration scheme to synthesize a path to the runway based on standard TRACON operations, aircraft state and type, and wind speed and direction.

Upon arrival into TRACON airspace, the FAST 4D Trajectory Generator predicts the arrival time of an aircraft at the final approach fix (outer marker) based on its current position, altitude, speed, and heading. The prediction is based on a set of standard arrival routes, air speed deceleration schedules, and altitude profiles that conform to standard operations at a given TRACON. The current implementation of FAST is based on Denver TRACON operations for arrivals to Stapleton International Airport. Next, the FAST 4D Trajectory Generator computes a range of arrival times based on the aircraft speed envelope and allowable path extension. These predicted trajectories are updated every 5 sec. If the STA and ETA are the same, the aircraft is maintained on its present nominal path, altitude, and speed profile to the runway. If the ETA shows the aircraft to be early, the FAST 4D Trajectory Generator will synthesize a descent trajectory that attempts to eliminate the time error by first decreasing the aircraft airspeed and then, if necessary, extending the path distance to the runway. If the ETA shows the aircraft to be late, the controller is advised to have the aircraft maintain higher speeds or shorten its path to the runway by utilizing the Horizontal Guidance Modes that will be described next.

Construction of the horizontal route always begins at the current position and heading of the aircraft and terminates at the final approach fix. The current position need not be on a standard path. The controller may vector the aircraft anywhere in the TRACON arrival airspace and a horizontal route will be synthesized based on either a route-intercept (RI) procedure or a waypoint capture (WC) procedure (refs. 3, 4).

Route intercept operates in conjunction with a set of standard or nominal arrival routes converging on the final approach course to the runway. The routes comprising the nominal arrival path from the north to Rwy 26L at Denver's Stapleton International Airport are the final approach course extending $15 \mathrm{n} . \mathrm{mi}$. beyond the outer marker (Altur), a base leg positioned 5.5 n.mi. from the outer marker and extending 15 n.mi. north from and perpendicular to the final approach course, and a downwind leg positioned $5 \mathrm{n} . \mathrm{mi}$. north of and parallel to the final approach course (fig. 1). Each route has a corridor width of \pm 1 n.mi. relative to its center line.

As an aircraft enters the TRACON airspace from one of the feeder gates (Drako or Keann) the FAST trajectory synthesis algorithm puts the aircraft into a free vector mode. In this mode, the algorithm seeks an interception of one of the defined route segments by extending the instantaneous heading vector. From the first point of interception, the algorithm completes the path by following along the nominal route to the final approach fix. After the aircraft has captured the downwind leg, the horizontal synthesis computes a new RI of the base leg. Similarly, once the aircraft has intercepted the base leg, a new RI of the final approach course is computed. The path to the runway is recomputed approximately every 5 sec based on the current position and heading. This free-vector mode with RI logic allows the controller the freedom to vector aircraft anywhere in the arrival airspace and still maintain a highly accurate estimate of arrival time as long as the aircraft is heading for a standard route segment.

The horizontal path synthesized by the waypoint capture (WC) mode consists of an initial circular arc starting at the current position and course followed by a straight-line segment leading directly to a designated capture waypoint, and ending with a circular arc turn intercepting the route containing the capture waypoint. The geometry of this construction is illustrated in figure 2 . The algorithm determines 
the radius of the turn from the airspeed, wind speed, and maximum allowable bank angle. Furthermore, the direction of the turn toward the capture waypoint is chosen so that the total length of the path is minimized. In order to compensate for computational delays and to allow for controller response time, the algorithm also moves the start of the turn at each computational cycle a distance equivalent to $10 \mathrm{sec}$ of flight time ahead of the current aircraft position. As in other trajectory synthesis modes, the predictive algorithm refreshes the WC profile in a 5-sec cycle using updated aircraft state information. The WC mode can be manually selected by the controller for special situations such as missed approach guidance. It is also selected automatically by FAST if the RI mode fails to generate a 4D trajectory under certain circumstances.

\section{Graphical Advisory Interface}

Similar to the Center DA, a vertical time line is used to display the current STA and ETA for all aircraft in, or expected to arrive in, the TRACON airspace.

The right side of the time line displays the current ETA for each aircraft in green. The left side of the time line displays the current STA for each aircraft in blue if arriving from the West and white if arriving from the East. This increases the speed with which the controller can correlate an aircraft's location on the time line with its location on the plan view display (PVD). If the STA and ETA are different during the aircraft's flight in the TRACON, FAST will provide speed advisories and heading vectors required for the aircraft to meet the STA. As the advisories are displayed, the ETA on the time line will adjust itself to reflect the effect of each update.

When FAST determines that a speed adjustment is necessary at a given point and the aircraft is within 5 n.mi. of that point, the advised Indicated Airspeed (IAS) is displayed on the aircraft data tag below the ground speed in orange. The use of color on the tag alerts the controller that an advisory is pending. Having the advised speed on the tag allows the controller to maintain his concentration on the aircraft position. In addition, the point along the current predicted path where the speed adjustment should be issued is highlighted with an orange marker to correlate with the orange speed advisory on the data tag. The 5-n.mi. advance notice and spatial display of the position at which the speed adjustment should occur allows the controller to plan ahead for its issuance.

Another common technique used by TRACON controllers to delay or advance an aircraft is to extend or compress the downwind leg of the approach path or vary the intercept of the final approach course. Thus, when an aircraft arrives from the West to land on Rwy $26 \mathrm{~L}$ and is within 5 n.mi. of its advised turn to base or turn to final, the data block is colored blue and a blue turn arc appears at the position where the instruction to turn should be issued. Once the aircraft has completed the base or final turn, the aircraft color reverts back to green, and the turn arc for that aircraft disappears. Similarly, aircraft arriving from the East are color-coded white for base and final turn advisories. The positions of the base and final turn advisories vary for each aircraft depending on its current time error relative to its STA and are displayed in the position that will eliminate the error.

In addition to its display on the time line, time error is also displayed below the altitude slot on the third line of each aircraft's data tag. The arrival time error, in seconds, is preceded either by an " $E$ " for early or an "L" for late. The controller may use this "Time Error" mode alone or in combination with the Speed/Vector and Time line advisory modes to improve time control accuracy. 


\section{SIMULATION DESCRIPTION}

A simulation evaluation of FAST, in conjunction with the TMA and DA, was conducted in January, 1990. Also included as one of the aircraft in the simulation was the Man-Vehicle Systems Research Facility (MVSRF) B727-200 full mission simulator. The objectives of the simulation were to 1) determine controller performance and runway capacity effects with and without automation tools, 2) evaluate controller acceptance of the FAST concept, 3) evaluate pilot acceptance of flying in the automation environment, and 4) determine the accuracy of the trajectory prediction algorithms in the TRACON.

A total of five TRACON controllers participated in the evaluation. Three controllers were from the Denver TRACON, and the other two were recently retired from the San Francisco Bay TRACON. Each controller participated for a period of one week. Typically, one day of training runs was necessary before data were taken. In the case of the Denver controllers, training was considered completed in one-half day. Data runs were started when both the experimenter and controller agreed that proficiency had reached a high level.

For the purposes described in this paper, two types of data runs were evaluated. The first was a baseline run in which the Center delivered traffic at the two Northern feeder gates, Drako and Keann, 7 n.mi. in-trail and the TRACON controller had no automation tools to assist in merging and spacing traffic. The second was a full automation run in which the Center delivered traffic to the feeder gates using the Center automation tools, DA and TMA. In all of the data runs presented in this paper, the arrival rate was an average of 43 aircraft per hour which provided a flow at maximum runway acceptance rate for single runway $26 \mathrm{~L}$ Instrument Flight Rules (IFR) operations at Denver. The arrival traffic rush lasted for $90 \mathrm{~min}$, contained $70 \%$ large aircraft and $30 \%$ heavy aircraft, and distributed traffic evenly $(50 \% / 50 \%)$ between the two arrival gates (Keann and Drako). Winds were calm. The 727 aircraft, which was piloted by active airline crews, participated up to four times in each simulation session. All other aircraft were "flown" by pseudo-pilots who used a keyboard to initiate changes in aircraft altitude, speed, and heading.

At the end of a simulation week, each controller was given a questionnaire and interviewed about the operational aspects of using the automation tools. Detailed results of these interviews, the pilot evaluations, and the accuracy data for the trajectory prediction algorithm will be presented in a later report.

\section{SIMULATION RESULTS}

Simulation results presented in this paper briefly address the issues of airspace utilization, interarrival spacings, capacity effects, and controller evaluations.

\section{Airspace Utilization}

One of the primary measures of an automation tool for final approach spacing is airspace utilization. The composite ground tracks of aircraft for the two types of runs discussed earlier, baseline and 
FAST+DA+TMA, are shown in figures 3 and 4. The figures superimpose the horizontal plane projections of the flight paths of all arrivals recorded during a typical simulation run. These figures show traffic arriving from both the northeast (Keann) and northwest (Drako) feeder gates. The runway is located in the southwest quadrant of these figures and is marked with an " $X$ ". The composite ground tracks in both of these figures resulted from the same list of input traffic covering a time range of slightly more than one hour of capacity limited flow (40-46 aircraft per hour). They are representative of all other runs made by the other controllers. In all runs, traffic was controlled by a single controller.

In the baseline run (fig. 3), the controller used considerably more airspace to merge and sequence traffic. By the end of the run, traffic had backed up such that he was turning the aircraft onto the final approach course $18 \mathrm{n} . \mathrm{mi}$. from the runway instead of the nominal $10 \mathrm{n} . \mathrm{mi}$. The length of the final approach allowed at Denver without having to coordinate with other controllers is approximately 20 n.mi. from the runway. In the automation run (fig. 4), almost all aircraft were turned to final at the nominal point between 10 and $11 \mathrm{n} . \mathrm{mi}$. from the runway. There were a few aircraft turned to base and final further out; however, this occurred at the advice of FAST in order to precisely alleviate potential conflicts and to build slots for aircraft which arrived in the TRACON off schedule. Although these aircraft were turned to base and final further from the runway, this did not cause a buildup in delay of trailing aircraft as would be the case in a manual system. Rather it served to alleviate a buildup in delay, and kept each trailing aircraft on its nominal and shortest turn to base and final paths. The ability of the automation tools to precisely expand and contract the base and turn to final points provides considerable advantages to the controller. Assisting the controllers in keeping most aircraft on a short final allows them plenty of airspace to expand in case of an overload of traffic. In the baseline run, if an overload of traffic were to arrive, the controller would soon be forced to use alternative procedures to control the traffic, such as holding, sending traffic upwind then downwind (i.e., from the northeast arrival stream to the downwind portion of the northwest arrival stream), or to shut off the Center traffic feed for several minutes.

\section{Interarrival Spacings}

Data were also recorded on interarrival spacing of aircraft for both the baseline and automation runs. Tables 2 and 3 present the results of all runs with capacity limited flow rate for all controllers. These tables present the sequence of aircraft ( $\mathrm{L}$ for large, $\mathrm{H}$ for heavy), mean interarrival distances at touchdown $(\overline{\mathrm{d}})$, one-sigma standard deviation of distance $\left(\sigma_{\mathrm{d}}\right)$, mean interarrival time at touchdown $(\overline{\mathrm{t}})$ and one-sigma standard deviation of time $\left(\sigma_{t}\right)$. As a point of reference, the desired distance separation for the LL and LH case is 3 n.mi., and the scheduling interval for this case was $78 \mathrm{sec}$. For the HL case, the desired distance separation is $5 \mathrm{n} . \mathrm{mi}$., and the scheduling interval was $125 \mathrm{sec}$. Although the controllers were instructed to adhere strictly to the FAST advisories, no data were deleted for the few cases when the controller missed or ignored the advisories.

Table 2 contains values measured for the baseline case which are very similar to those measured for the manual system in reference 1 . The tables show a substantial decrease in interarrival spacing in both distance and time. The automation tool runs resulted in a decrease in mean distance separation of $0.4 \mathrm{n}$.mi. and a decrease in mean time separation of $9.8 \mathrm{sec}$ for the LL and LH case. Most significant is the decrease in the standard deviations of both distance and time separations seen in the tables. Similar results are seen for the HL case. 


\section{Capacity Effects}

Based on these results, increased capacity can be estimated. If all aircraft were "large," the runway capacity for this simulation based on the scheduling interval of $78 \mathrm{sec}$ would be 46.2 aircraft per hour. In the baseline runs, controllers delivered traffic at a rate of 38.8 aircraft per hour, based on the mean time separation. For the automation runs, the arrival rate was 43.4 aircraft per hour. This implies a capacity increase of approximately 4.6 aircraft/hour in the automation runs over the baseline runs.

An alternate method for estimating the capacity increase for the automation system is to make use of the empirically determined standard deviations in arrival time error. In this method a time buffer is added to the minimum separation times such that all aircraft arriving within one sigma of the scheduling interval do not violate the minimum separation time standards (i.e., $78 \mathrm{sec}$ ). It can be shown that the gain in arrival rate obtained by this method is 4.6 aircraft per hour, which is consistent with the previous method. It should be noted that such an increase in landing rate, if realized in practice, would produce substantial delay reductions during rush periods.

\section{Controller Evaluations}

As described earlier, the controllers were given a questionnaire and interviewed at the conclusion of each simulation week. A full analysis of responses to the questionnaire will be presented in a future report; however, some discussion of the general trends follows. The most important response was the strong agreement among all controllers that workload was reduced. This reduction in workload was manifested by a reduction of the number of speed and heading clearances issued for each aircraft, as well as a perceived reduction in mental workload. Controllers found the time line useful for both sequence and schedule information. They said that the turn and speed advisories were easy to see, provided sufficient time to issue them, and usually coincided with what they would have done in sequencing aircraft. The questionnaire also showed that the speed and vector advisories were their favorite feature. When the advisories did not coincide with their own plan, they commented that the FAST generated plan was just as good and sometimes better. They did not find that additional vectoring was necessary beyond the FAST advisories, and they thought the tools were flexible and did not feel restricted in their own decision making.

Several suggestions were made for improving the controller interface though none of the suggestions pointed to basic changes or major additional requirements in the interface design. Some controllers suggested a "distance-based time line" on which in-trail distance projected at the runway is displayed rather than time. Such a method has been used in the Center DA tool and could be adapted to the TRACON. Another suggestion was to give the controller an option to position the nominal downwind and base leg at his or her discretion, and to incorporate certain controller preferences in the advisory logic. These and other suggestions are being considered for incorporation into FAST.

Finally, all of the controllers sxpressed strong support for the integrated terminal automation system concept composed on Certer DA and TMA and TRACON FAST. In particular, the Denver TRACON contrullers were especially enthusiastic in their support of FAST and were eager to participate in further estim 2 ant evaluatior: 


\section{CONCLUDING REMARKS}

The automation tools described in this paper and evaluated in the simulation were designed primarily for TRACON controllers. However, the Center automation tools that were used to feed traffic into the TRACON played an important role in the success of the TRACON tools. The Center tools were effective in delivering traffic to the feeder gates well sorted and with little time error, thus simplifying the TRACON controller's job with or without TRACON tools. Therefore, a total systems approach that integrates Center and TRACON automation tools is clearly the best method to increase efficiency.

The simulation evaluation of the Final Approach Spacing Tool (FAST) demonstrated efficient airspace utilization and reduced interarrival separations, and resulted in strong controller acceptance of the TRACON automation tools. With FAST, controllers were consistently able to maintain final approach intercepts of 10 to $11 \mathrm{n} . \mathrm{mi}$. from the runway for over an hour of runway-capacity-limited arrival traffic. Without the automation tools, final approach intercepts were expanded to 18 to $20 \mathrm{n}$.mi. In addition, the mean interarrival separations were reduced by $0.4 \mathrm{n} . \mathrm{mi}$. or $9 \mathrm{sec}$. This reduction in separation translates to an increase in landing rate of 4.6 aircraft per hour for a single runway in IFR conditions. Finally, all of the controllers felt there was a significant decrease in workload which was manifested by a perceived reduction in clearances as well as a perceived reduction in mental workload.

Further simulation evaluations of FAST are planned in the near future. These will address such issues as testing FAST standalone without the Center automation tools, DA and TMA, and under varying wind conditions. Ultimately, however, a test of the concept at the Denver TRACON or a similar facility must be conducted in order to establish the effectiveness of the tools with a high level of confidence. 


\section{REFERENCES}

1. Martin, D. A.; and Willett, F. M.: Development and Application of a Terminal Spacing System. Rep. No. NA-68-25 (RD-68-16), Federal Aviation Administration, Aug. 1968.

2. Credeur, L.; and Capron, W. R.: Simulation Evaluation of TIMER, a Time-Based, Terminal Air Traffic, Flow Management Concept. NASA TP-2870, Feb. 1989.

3. Erzberger, H.; and Nedell, W.: Design of Automated System for Management of Arrival Traffic. NASA TM-102201, June 1989.

4. Davis, T. J.; Erzberger, H.; and Bergeron, H.: Design of a Final Approach Spacing Tool for TRACON Air Traffic Control. NASA TM-102229, Sept. 1989.

5. Neuman, F.; and Erzberger, H.: Analysis of Sequencing and Scheduling Methods for Arrival Traffic. NASA TM-102795, April 1990.

6. Tobias, L.: Time Scheduling of a Mix of 4D Equipped and Unequipped Aircraft. Proceedings of 22nd IEEE Conference on Decision and Control, San Antonio, TX, Dec. 1983, pp. 483-488. 
Table 1. Separation Distances

\begin{tabular}{|c|c|c|c|c|}
\hline \multirow{5}{*}{ 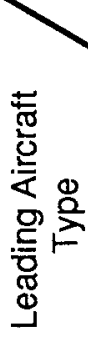 } & & \multicolumn{3}{|c|}{ Trailing Aircraft Type } \\
\hline & & Heavy & Large & Light \\
\hline & Heavy & 4 & 5 & 6 \\
\hline & Large & 3 & 3 & 4 \\
\hline & Light & 3 & 3 & 3 \\
\hline
\end{tabular}

Table 2. Interarrival Data for Baseline Runs

\begin{tabular}{cccccc}
\hline $\begin{array}{c}\text { Aircraft } \\
\text { Sequence }\end{array}$ & $\begin{array}{c}\text { Number of } \\
\text { Occurrences }\end{array}$ & $\begin{array}{c}\overline{\mathrm{d}} \\
\text { (n.mi.) }\end{array}$ & $\begin{array}{c}\sigma_{\mathrm{d}} \\
\text { (n.mi.) }\end{array}$ & $\begin{array}{c}\overline{\mathrm{t}} \\
(\mathrm{sec})\end{array}$ & $\begin{array}{c}\sigma_{\mathrm{t}} \\
(\mathrm{sec})\end{array}$ \\
\hline LL and LH & 83 & 3.8 & 1.0 & 92.8 & 23.9 \\
HL & 21 & 5.6 & 1.5 & 127.8 & 29.8 \\
\hline
\end{tabular}

Table 3. Interarrival Data for Automation Runs

\begin{tabular}{cccccc}
\hline $\begin{array}{c}\text { Aircraft } \\
\text { Sequence }\end{array}$ & $\begin{array}{c}\text { Number of } \\
\text { Occurrences }\end{array}$ & $\begin{array}{c}\overline{\mathrm{d}} \\
\text { (n.mi.) }\end{array}$ & $\begin{array}{c}\sigma_{\mathrm{d}} \\
\text { (n.mi.) }\end{array}$ & $\begin{array}{c}\overline{\mathrm{t}} \\
(\mathrm{sec})\end{array}$ & $\begin{array}{c}\sigma_{\mathrm{t}} \\
(\mathrm{sec})\end{array}$ \\
\hline LL and LH & 125 & 3.4 & 0.7 & 83.0 & 17.0 \\
HL & 30 & 5.4 & 0.9 & 124.5 & 16.7 \\
\hline
\end{tabular}




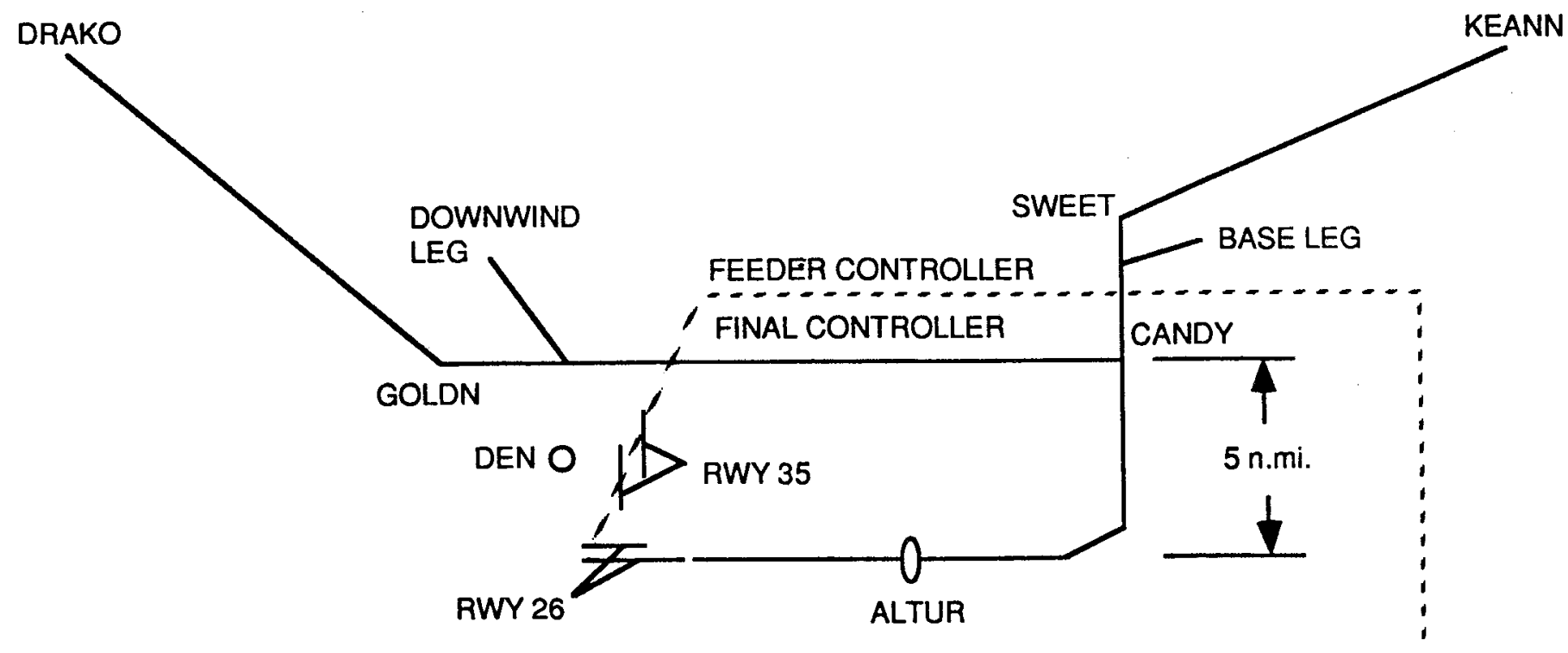

Figure 1. Arrival procedure for Denver TRACON to Rwy 26L.

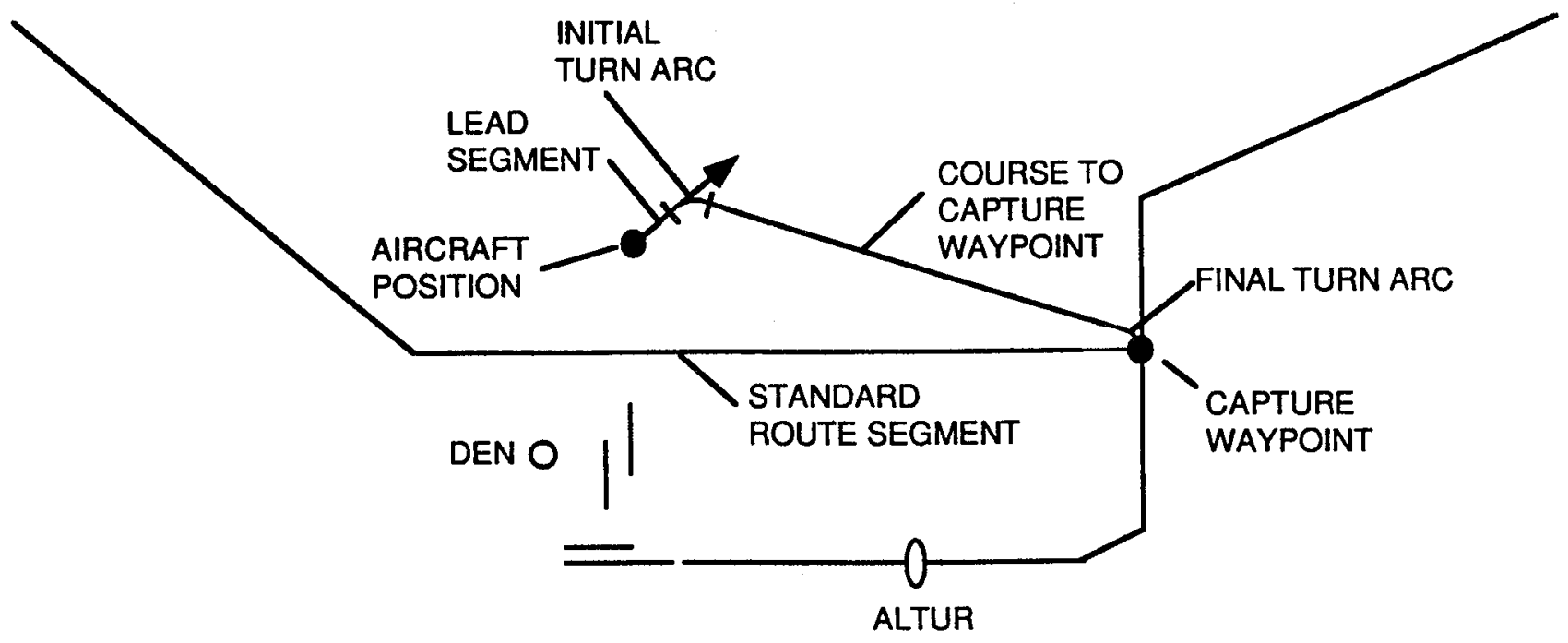

Figure 2. Waypoint capture guidance. 


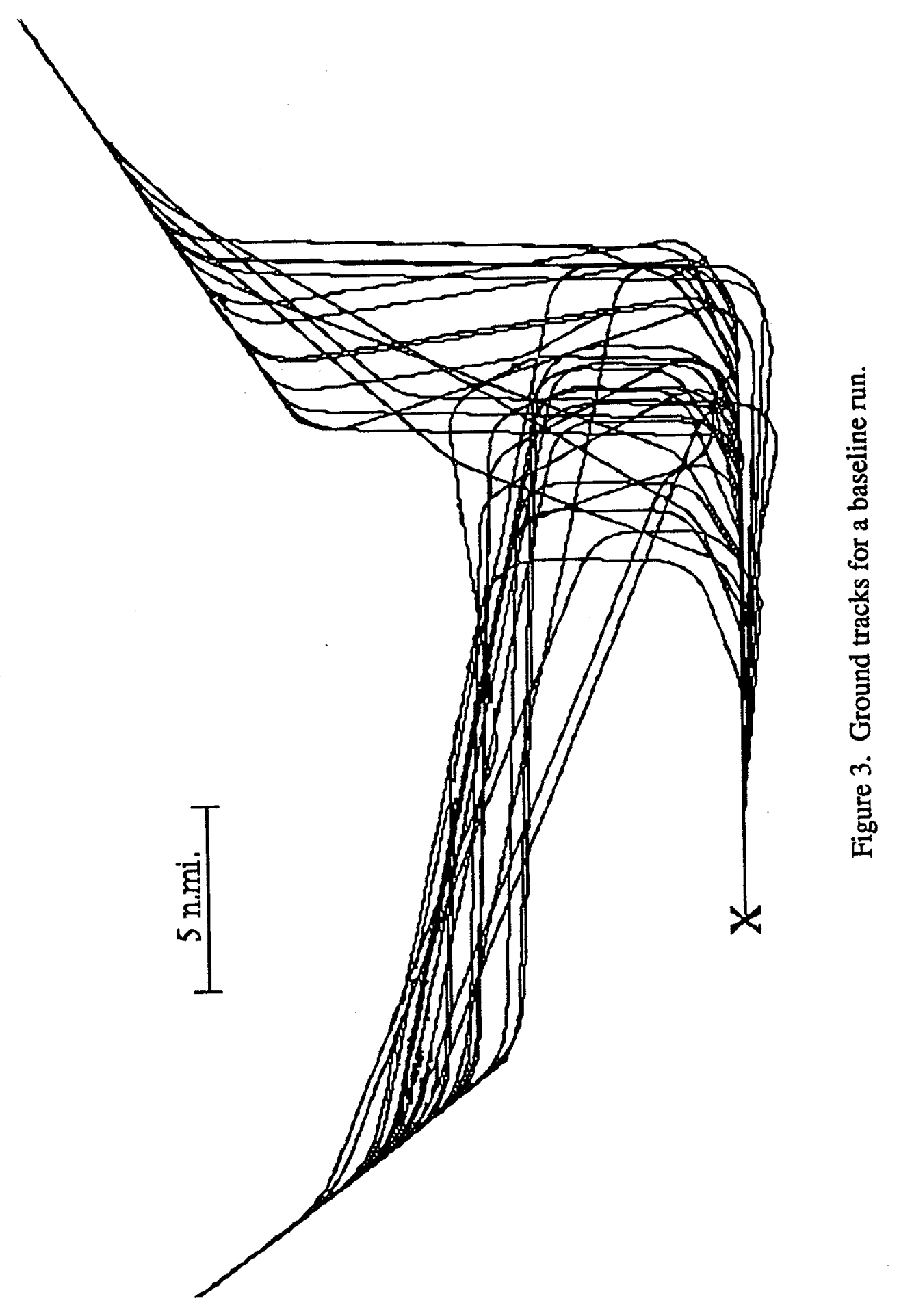




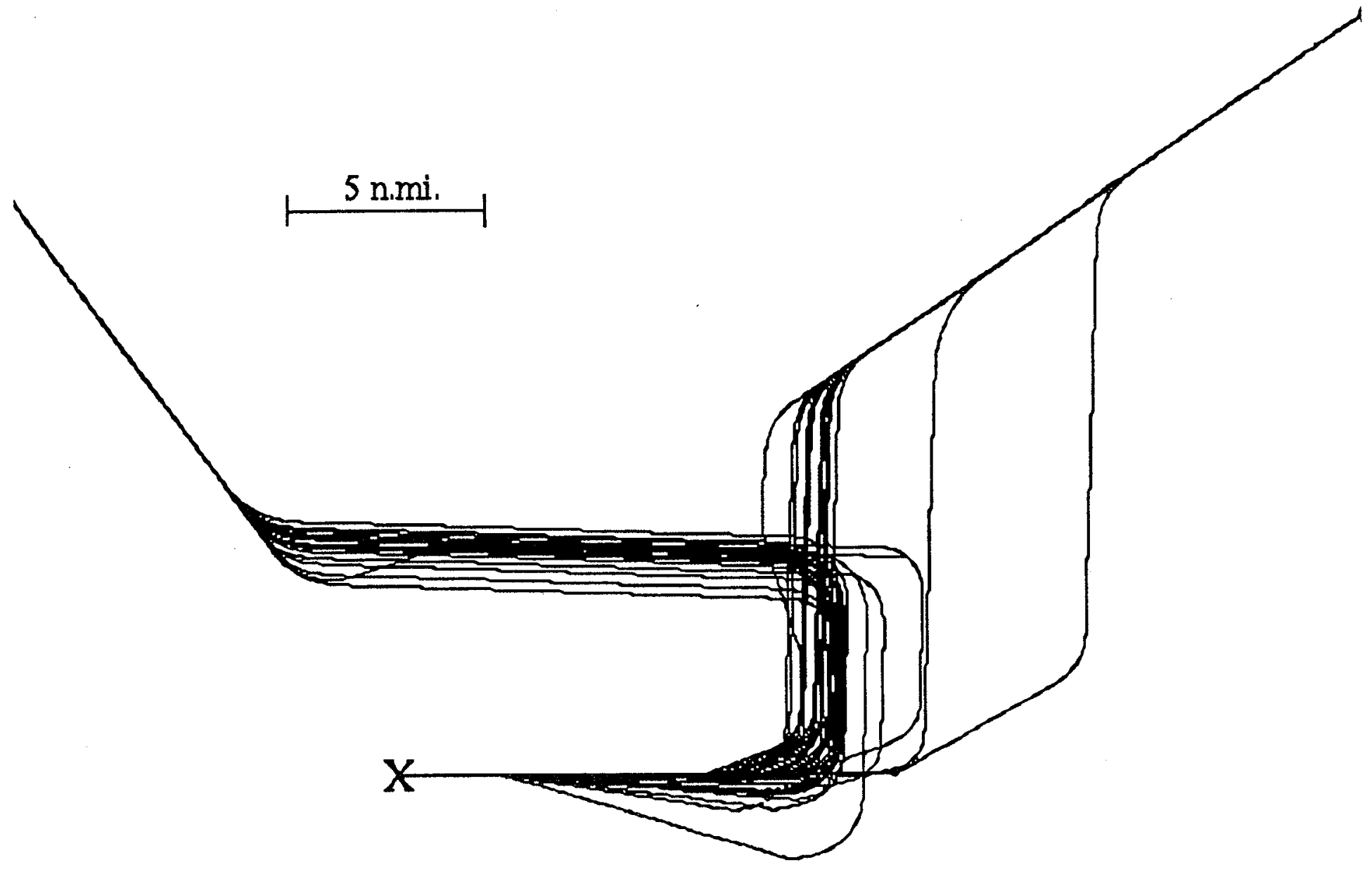

Figure 4. Ground tracks for an automation run. 


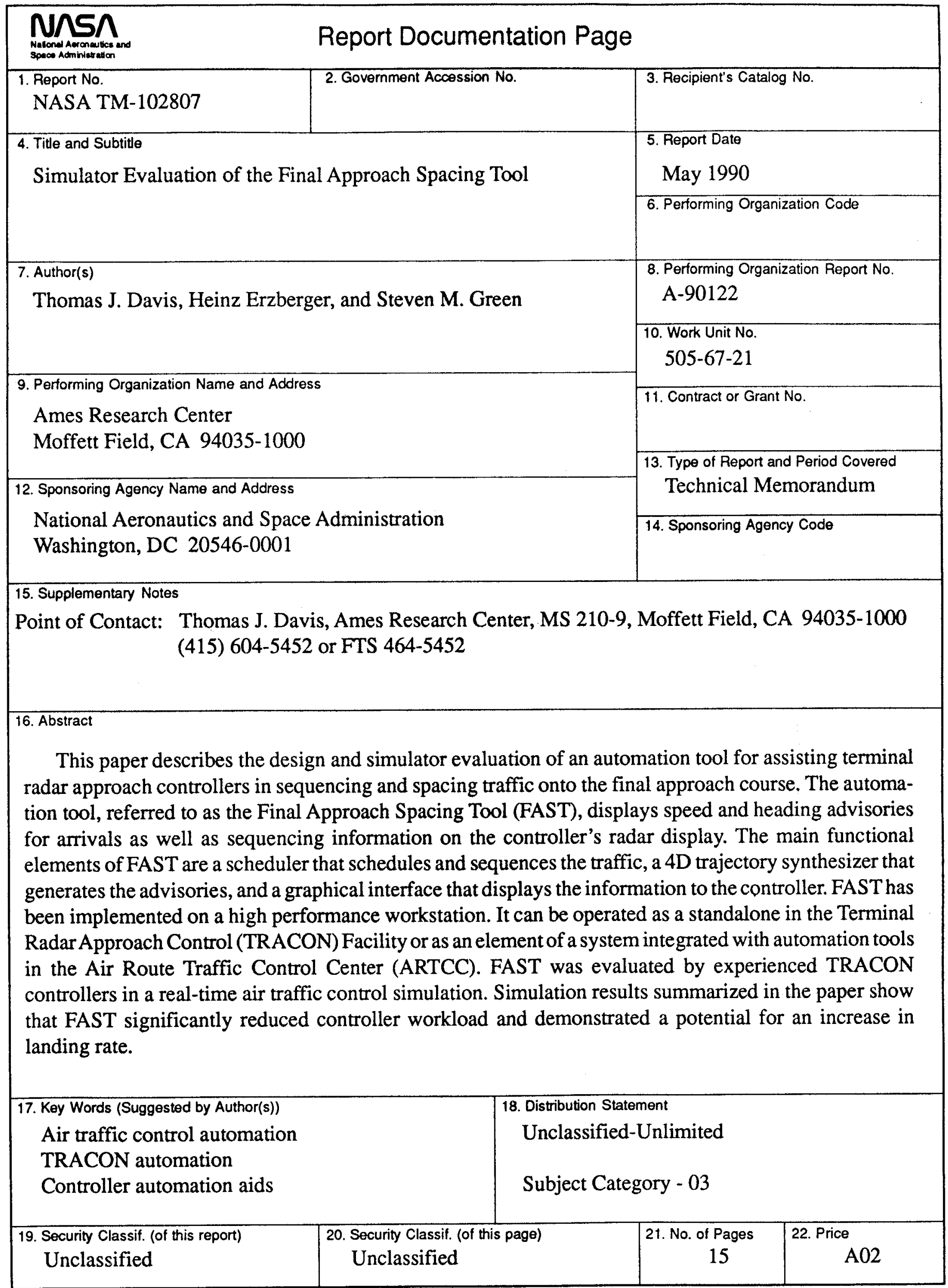


